\title{
Universal results from an alternate random matrix model for QCD with a baryon chemical potential
}

\author{
James C. Osborn \\ Physics Department, University of Utah, Salt Lake City, UT 84112, USA
}

\begin{abstract}
We introduce a new non-Hermitian random matrix model for QCD with a baryon chemical potential. This model is a direct chiral extension of a previously studied model that interpolates between the Wigner-Dyson and Ginibre ensembles. We present exact results for all eigenvalue correlations for any number of quark flavors using the orthogonal polynomial method. We also find that the parameters of the model can be scaled to remove the effects of the chemical potential from all thermodynamic quantities until the finite density phase transition is reached. This makes the model and its extensions well suited for studying the phase diagram of QCD.
\end{abstract}

Random matrix models have long been used as effective models for a variety of complex quantum systems. More recently the class of non-Hermitian random matrix models has received much attention in systems including the fractional quantum hall effect [1], two dimensional charged plasmas 2] and quantum chaotic scattering (see 3] for a review). Additionally one was introduced by Stephanov as a model for QCD with a baryon chemical potential [4]. Since then this model and related extensions have often been used to study the phase diagram of finite density QCD [5]. It is also useful for evaluating numerical algorithms that might be used to simulate finite density QCD [ 6 ] and in studying the density of the eigenvalues of the Dirac operator 7]. Despite the success of this model, exact results for the eigenvalue correlations have remained notably absent. Only recently has the eigenvalue density been obtained in the low energy (microscopic) limit by Splittorff and Verbaarschot [8].

Another model for the eigenvalues of the QCD Dirac operator with a chemical potential was proposed by Akemann [9]. This model was constructed as a chiral extension of a previously studied non-Hermitian matrix model. The main attraction of this model is that it can be solved exactly for all the eigenvalue correlations. However, the model is defined only in terms of the eigenvalues, and not from a matrix model based on the symmetries of the Dirac operator with a chemical potential. It was later shown that the partition function for this model agreed with that of Stephanov's in the microscopic limit 10]. This agreement, however, only holds for a sufficiently small chemical potential as can be seen clearly by comparing the microscopic spectral density [8].

Here we will consider a new matrix model for QCD with a baryon chemical potential which is also based on the form of the Dirac operator like in Stephanov's model, but can be solved for all spectral correlations like the model of Akemann. We will give exact results for the correlations with any number of quark flavors. We will also consider the results in the microscopic limit where the correlations should agree with QCD due to universality. Lastly we look at the partition function and show that one can absorb the effects of the chemical potential by scaling the parameters of the model. This then provides a physically accurate model that shows no change in thermodynamic quantities until the finite density phase transition is reached.

The matrix model originally studied by Stephanov is

$$
\mathcal{D}_{I}=\left(\begin{array}{cc}
0 & i A+\mu \\
i A^{\dagger}+\mu & 0
\end{array}\right)
$$

with $A$ a random complex matrix and $\mu$ is multiplied by the identity. This is based on the form of the Dirac operator $\not D+\mu \gamma_{0}$ in a chiral basis. Here the matrix elements of $\gamma_{0}$ are chosen to be constant which is accurate when $\mu$ is very large, but it makes the model difficult to solve.

Here we consider an alternate basis where the matrix elements of $\gamma_{0}$ are not taken as constant but are modeled with random matrices as

$$
\mathcal{D}_{I I}=\left(\begin{array}{cc}
0 & i A+\mu B \\
i A^{\dagger}+\mu B^{\dagger} & 0
\end{array}\right) .
$$

Here $A$ and $B$ are complex $(N+\nu) \times N$ matrices with $\nu$ the topological charge. The QCD partition function with $N_{f}$ quark flavors can now be modeled as

$$
Z=\mathcal{N} \int d A d B w_{G}(A) w_{G}(B) \prod_{j=1}^{N_{f}} \operatorname{det}\left(\mathcal{D}_{I I}+m_{j}\right)
$$

where $\mathcal{N}$ is a normalization constant we will chose later. At low energies the results are universal and do not depend on the choice of the distribution for the matrix elements. We therefore make the simple choice of a Gaussian measure given by

$$
w_{G}(A)=\exp \left(-\alpha N \operatorname{Tr} A^{\dagger} A\right) .
$$

At $\mu=0$ the parameter $\sqrt{\alpha}=\Sigma V / 2 N$ is determined by the absolute value of the chiral condensate in the chiral limit $(\Sigma)$ and the space-time volume $(V)$. We will consider the $\mu$ dependence of $\alpha$ later. For now we will treat it as a constant and calculate the distribution of eigenvalues.

In order to diagonalize the Dirac matrix we first make the substitutions $C=i A+\mu B$ and $D=i A^{\dagger}+\mu B^{\dagger}$. The 
partition function becomes

$$
Z=\mathcal{N}^{\prime} \int d C d D w_{2}(C, D) \prod_{j=1}^{N_{f}} \operatorname{det}\left(\begin{array}{cc}
m_{j} & C \\
D & m_{j}
\end{array}\right)
$$

with $\mathcal{N}^{\prime}$ a $\mu$ dependent constant and the weight is

$$
\begin{aligned}
w_{2}(C, D)=\exp [ & -\frac{\alpha N\left(1+\mu^{2}\right)}{4 \mu^{2}} \operatorname{Tr}\left(C^{\dagger} C+D^{\dagger} D\right) \\
& \left.-\frac{\alpha N\left(1-\mu^{2}\right)}{4 \mu^{2}} \operatorname{Tr}\left(C D+C^{\dagger} D^{\dagger}\right)\right] .
\end{aligned}
$$

The matrix $\mathcal{D}_{I I}$ has exactly $\nu$ zero eigenvalues and $N$ pairs of eigenvalues which we write as $\pm i z_{k}$. The nonzero eigenvalues of $C D$ and $D C$ are then equal to $-z_{k}^{2}$. We now introduce the parameterization

$$
\begin{aligned}
& C=U(X+R) V \\
& D=V^{\dagger}(Y+S) U^{\dagger}
\end{aligned}
$$

where $U$ and $V$ are unitary square matrices, $X$ and $R$ are complex $(N+\nu) \times N$ and $Y$ and $S$ complex $N \times$ $(N+\nu)$ matrices. The elements $X_{i j}$ and $Y_{i j}$ are zero except when $i=j$ and $R_{i j}$ and $S_{i j}$ are zero except when $i<j$. The above decomposition is not unique and we must restrict the integration measure on $U$ to the group $\mathrm{U}(N+\nu) /\left[\mathrm{U}(1)^{N} \otimes \mathrm{U}(\nu)\right]$ and on $V$ to $\mathrm{U}(N) / \mathrm{U}(1)^{N}$. The Jacobian for this transformation can be calculated

$$
\left|\Delta\left(z^{2}\right)\right|^{2} \prod_{k}\left|x_{k}\right|^{2 \nu}
$$

where $\Delta\left(z^{2}\right)=\prod_{i<j}\left(z_{i}^{2}-z_{j}^{2}\right)$ is the Vandermonde determinant. Here we have used that $x_{k} y_{k}=-z_{k}^{2}$ with $x_{k}$ and $y_{k}$ the diagonal elements of $X$ and $Y$ respectively. With this parameterization the integrals over $U, V, R$ and $S$ become trivial and we are left with integrals over the complex numbers $x_{k}$ and $y_{k}$. We can change the integral over $y_{k}$ to an integral over the eigenvalues $z_{k}$ and then integrate out $x_{k}$ to arrive at the form

$$
Z=\mathcal{N}^{\prime \prime} \prod_{j=1}^{N_{f}} m_{j}^{\nu} \int\left|\Delta\left(z^{2}\right)\right|^{2} \prod_{k=1}^{N} w\left(z_{k}\right) d z_{k} \prod_{j=1}^{N_{f}}\left(z_{k}^{2}+m_{j}^{2}\right)
$$

with $\mathcal{N}^{\prime \prime}$ another normalization constant and

$$
\begin{aligned}
w(z) & =|z|^{2 \nu+2} \exp \left(\frac{\alpha N\left(1-\mu^{2}\right)}{4 \mu^{2}}\left(z^{2}+z^{* 2}\right)\right) \\
& \times K_{\nu}\left(\frac{\alpha N\left(1+\mu^{2}\right)}{2 \mu^{2}}|z|^{2}\right)
\end{aligned}
$$

where $K_{\nu}$ is a modified Bessel function.

The eigenvalue representation of the partition function (9) can be related to the model considered previously by Akemann. For large argument the Bessel function becomes $K_{\nu}(x) \approx \sqrt{\pi / 2 x} \exp (-x)$ which gives

$$
\begin{aligned}
w(z) & \approx \sqrt{\frac{\pi \mu^{2}}{\alpha N\left(1+\mu^{2}\right)}}|z|^{2 \nu+1} \\
& \times \exp \left(-\frac{\alpha N\left(1+\mu^{2}\right)}{2 \mu^{2}}\left[|z|^{2}-\frac{\tau}{2}\left(z^{2}+z^{* 2}\right)\right]\right)
\end{aligned}
$$

where we have set $\tau=\left(1-\mu^{2}\right) /\left(1+\mu^{2}\right)$. This is the same model considered in [9]. The two models agree when

$$
\alpha N\left(1+\mu^{2}\right)\left|z_{m i n}\right|^{2} \gg 2 \mu^{2} .
$$

The smallest eigenvalue $z_{\min }$ has an average size around $\pi /(\Sigma V)$ at $\mu=0$. It is then convenient to consider the above condition in the microscopic 11] and weak nonHermiticity [12] limits given by taking $N \rightarrow \infty$ while holding $\eta_{k}=\Sigma V z_{k}, \mu_{s}^{2}=2 N \mu^{2}$ and $\alpha$ fixed. The above condition then becomes

$$
\left|\eta_{\min }\right|^{2} \gg 4 \mu_{s}^{2}
$$

In the microscopic limit the two models agree only for sufficiently small $\mu_{s}$. Since the new model is based directly on the Dirac operator we expect that (10) is the correct eigenvalue weight for QCD at low energies even for larger values of $\mu_{s}$.

\section{QUENCHED SPECTRUM}

Given the expression for the partition function in terms of the eigenvalues of the Dirac operator we can now solve for their correlations. This was done in [9] for the model with weight (11) at $N_{f}=0$ using the method of orthogonal polynomials extended to the complex plane. There it was shown that the corresponding orthogonal polynomials are the Laguerre polynomials. From that all spectral correlations can be determined analytically. It turns out that the orthogonal polynomials corresponding to the weight (10) are also Laguerre. We can therefore proceed in similar fashion.

One can easily verify that the monic polynomials

$$
p_{k}^{0}(z)=\left(\frac{\mu^{2}-1}{\alpha N}\right)^{k} k ! L_{k}^{\nu}\left(\frac{\alpha N z^{2}}{1-\mu^{2}}\right)
$$

are orthogonal with respect to the integral

$$
\int_{\operatorname{Re}(z)>0} p_{k}^{0}(z) p_{\ell}^{0}\left(z^{*}\right) w(z) d z=h_{k}^{0} \delta_{k \ell}
$$

with

$$
h_{k}^{0}=\frac{\pi \mu^{2}\left(1+\mu^{2}\right)^{2 k+\nu} k !(k+\nu) !}{2(\alpha N)^{2 k+\nu+2}} .
$$

Here we restricted the range of integration to the positive real half of the complex plane. This avoids double counting pairs of eigenvalues with opposite signs and ensures agreement with previously obtained results at $\mu=0$. 
The $n$-point spectral correlator can be written as

$$
\rho_{N}^{0}\left(z_{1}, \ldots, z_{n}\right)=\operatorname{det}_{1 \leq i, j \leq n}\left[\mathcal{K}_{N}^{0}\left(z_{i}, z_{j}\right)\right]
$$

in terms of the kernel

$$
\mathcal{K}_{N}^{0}(x, y)=\sqrt{w(x) w(y)} \sum_{k=0}^{N-1} \frac{1}{h_{k}^{0}} p_{k}^{0}(x) p_{k}^{0}\left(y^{*}\right) .
$$

We can also evaluate the result in the microscopic limit. The microscopic limit of the $n$-point correlator is

$$
\rho_{s}^{0}\left(\eta_{1}, \ldots, \eta_{n}\right)=\lim _{N \rightarrow \infty} \frac{1}{(\Sigma V)^{2 n}} \rho_{N}\left(\frac{\eta_{1}}{\Sigma V}, \ldots, \frac{\eta_{n}}{\Sigma V}\right)(19)
$$

with $\alpha$ kept fixed. This limit can be taken following the procedure in [9]. The result can be written as a determinant similar to (17) but with the microscopic kernel

$$
\begin{aligned}
\mathcal{K}_{s}^{0}(x, y) & =\frac{|x y|^{\nu+1}}{4 \pi \mu_{s}^{2}\left(x y^{*}\right)^{\nu}} \sqrt{K_{\nu}\left(\frac{|x|^{2}}{4 \mu_{s}^{2}}\right) K_{\nu}\left(\frac{|y|^{2}}{4 \mu_{s}^{2}}\right)} \\
& \times \mathrm{e}^{\frac{\operatorname{Re}\left(x^{2}+y^{2}\right)}{8 \mu_{s}^{2}}} \int_{0}^{1} \mathrm{e}^{-2 \mu_{s}^{2} t} J_{\nu}(x \sqrt{t}) J_{\nu}\left(y^{*} \sqrt{t}\right) d t
\end{aligned}
$$

The spectral density is obtained by setting $x=y$ in the above expression and agrees with the result given in 8 based on the model using (1). This agreement is expected since both models are based directly on the form of the Dirac operator. Only the microscopic results are universal, though, and the macroscopic correlations of the two models do not necessarily agree.

\section{UNQUENCHED SPECTRUM}

We can also extend the results to an arbitrary number of flavors using an iterative procedure similar to the one used for the case of $\mu=0$ in [13]. However since the mass term is only a function of $z$ and not $z^{*}$ we can't use a single set of orthogonal polynomials. Instead we construct a set of biorthogonal polynomials for $a$ flavors that satisfy the biorthogonality condition

$$
\int_{\operatorname{Re}(z)>0} p_{k}^{a}(z) q_{l}^{a}\left(z^{*}\right) w(z) \prod_{j=1}^{a}\left(z^{2}+m_{j}^{2}\right) d z=h_{k}^{a} \delta_{k l}
$$

The monic polynomials can be defined iteratively by

$$
\begin{aligned}
p_{k}^{a}(z) & =\frac{p_{k+1}^{a-1}(z) p_{k}^{a-1}\left(i m_{a}\right)-p_{k}^{a-1}(z) p_{k+1}^{a-1}\left(i m_{a}\right)}{p_{k}^{a-1}\left(i m_{a}\right)\left(z^{2}+m_{a}^{2}\right)} \\
q_{k}^{a}\left(z^{*}\right) & =\frac{h_{k}^{a-1}}{p_{k}^{a-1}\left(i m_{a}\right)} \sum_{n=0}^{k} \frac{p_{n}^{a-1}\left(i m_{a}\right)}{h_{n}^{a-1}} q_{n}^{a-1}\left(z^{*}\right)
\end{aligned}
$$

with the convention that $q_{n}^{0}\left(z^{*}\right)=p_{n}^{0}\left(z^{*}\right)$. The normalization coefficient can also be derived recursively by

$$
h_{k}^{a}=-\frac{p_{k+1}^{a-1}\left(i m_{a}\right)}{p_{k}^{a-1}\left(i m_{a}\right)} h_{k}^{a-1} .
$$

An explicit formula for the polynomials for a general weight function appears in 14].

For convenience we will define the function

$$
\phi_{k}^{a}(z)=p_{k}^{a}(z) \prod_{j=1}^{a}\left(z^{2}+m_{j}^{2}\right)
$$

The $a$ flavor kernel can now be written as

$$
\mathcal{K}_{N}^{a}(x, y)=\tilde{\mathcal{K}}_{N}^{a}(x, y) \sqrt{w(x) w(y)} \prod_{j=1}^{a} \sqrt{\frac{y^{2}+m_{j}^{2}}{x^{2}+m_{j}^{2}}}
$$

with

$$
\tilde{\mathcal{K}}_{N}^{a}(x, y)=\sum_{k=0}^{N-1} \frac{1}{h_{k}^{a}} \phi_{k}^{a}(x) q_{k}^{a}\left(y^{*}\right)
$$

We can get a recursion relation for this by substituting (22), (23) and (24) into (26) and rearranging the sums as

$$
\tilde{\mathcal{K}}_{N}^{a}(x, y)=\tilde{\mathcal{K}}_{N}^{a-1}(x, y)-\frac{\phi_{N}^{a-1}(x)}{\phi_{N}^{a-1}\left(i m_{a}\right)} \tilde{\mathcal{K}}_{N}^{a-1}\left(i m_{a}, y\right) .
$$

Repeated application of these relations gives an expression for the $a$ flavor kernel in terms of the zero flavor kernel and the functions $p_{N+k}^{0}(x)$ for $0 \leq k \leq a-1$. Notice that the modified kernel $\tilde{\mathcal{K}}_{N}^{a}(x, y)$ vanishes at $x=i m_{j}$ for $0 \leq j \leq a$. It can therefore be written in the form of a determinant

$\tilde{\mathcal{K}}_{N}^{a}(x, y)=\frac{\left|\begin{array}{cccc}\tilde{\mathcal{K}}_{N}^{0}(x, y) & p_{N}^{0}(x) & \cdots & p_{N+a-1}^{0}(x) \\ \tilde{\mathcal{K}}_{N}^{0}\left(i m_{1}, y\right) & p_{N}^{0}\left(i m_{1}\right) & \cdots & p_{N+a-1}^{0}\left(i m_{1}\right) \\ \vdots & \vdots & \ddots & \vdots \\ \tilde{\mathcal{K}}_{N}^{0}\left(i m_{a}, y\right) & p_{N}^{0}\left(i m_{a}\right) & \cdots & p_{N+a-1}^{0}\left(i m_{a}\right)\end{array}\right|}{\left|p_{N+\ell-1}^{0}\left(i m_{k}\right)\right|_{1 \leq k, \ell \leq a}}$.

The denominator was determined by requiring that the coefficient of $\tilde{\mathcal{K}}_{N}^{0}(x, y)$ is unity.

We can also take the microscopic limit of the above kernel. For this we also scale the masses with the inverse of the volume keeping $\hat{m}_{k}=\Sigma V m_{k}$ fixed. This limit must be taken carefully and can be done with a procedure similar to that in [15]. The result is

$$
\mathcal{K}_{s}^{a}(x, y)=\mathcal{K}_{s}^{0}(x, y) \frac{\tilde{\mathcal{K}}_{s}^{a}(x, y)}{\tilde{\mathcal{K}}_{s}^{0}(x, y)} \prod_{j=1}^{a} \sqrt{\frac{y^{2}+\hat{m}_{j}^{2}}{x^{2}+\hat{m}_{j}^{2}}}
$$

with $\tilde{\mathcal{K}}_{s}^{a}(x, y)$ equal to

$$
\frac{\mid \begin{array}{cccc}
\tilde{\mathcal{K}}_{s}^{0}(x, y) & J_{\nu}(x) & \cdots & x^{a-1} J_{\nu+a-1}(x) \\
\tilde{\mathcal{K}}_{s}^{0}\left(i \hat{m}_{1}, y\right) & J_{\nu}\left(i \hat{m}_{1}\right) & \cdots & \left(i \hat{m}_{1}\right)^{a-1} J_{\nu+a-1}\left(i \hat{m}_{1}\right) \\
\vdots & \vdots & \ddots & \vdots \\
\tilde{\mathcal{K}}_{s}^{0}\left(i \hat{m}_{a}, y\right) & J_{\nu}\left(i \hat{m}_{a}\right) & \cdots & \left(i \hat{m}_{a}\right)^{a-1} J_{\nu+a-1}\left(i \hat{m}_{a}\right)
\end{array}}{\left|\left(i \hat{m}_{k}\right)^{\ell-1} J_{\nu+\ell-1}\left(i \hat{m}_{k}\right)\right|_{1 \leq k, \ell \leq a}}
$$


The function $\tilde{\mathcal{K}}_{s}^{0}(x, y)$ is defined as the integral that appears in (20). This gives the general result for the microscopic spectral correlations of the QCD Dirac operator with a baryon chemical potential.

\section{PARTITION FUNCTION}

This model can also be used as an effective model for studying qualitative properties of the finite density QCD phase diagram. We can construct this model so that it has the correct physical behavior below the finite density phase transition. To see this we will look at the partition function which is simply given by

$$
Z_{N}^{N_{f}}=\mathcal{N}^{\prime \prime} N ! \prod_{j=1}^{N_{f}} m_{j}^{\nu} \prod_{k=0}^{N-1} h_{k}^{N_{f}}
$$

Using the recursion relations (23) and (22) the partition function can be written in terms of the quenched polynomials (14). The result is

$$
Z_{N}^{N_{f}}=\mathcal{N}_{N}^{N_{f}} \prod_{j=1}^{N_{f}} m_{j}^{\nu} \frac{\operatorname{det}_{1 \leq k, \ell \leq N_{f}}\left[p_{N+\ell-1}^{0}\left(i m_{k}\right)\right]}{\Delta\left(m^{2}\right)}
$$

This result can also be obtained from the more general expression given in [16].

Notice that the polynomials $p_{k}^{0}$ only depend on $\mu$ through the combination $\alpha /\left(1-\mu^{2}\right)$. If we had started with a $\mu$ dependent $\alpha=\left(1-\mu^{2}\right) \alpha_{0}$ and an appropriate normalization $\mathcal{N}$ then we could have made a partition function that doesn't depend on $\mu$. This means that all thermodynamic quantities would remain at their $\mu=0$ values for $\mu<1$. This agrees with the phenomenological expectation that the baryon number density should remain at zero until a first order phase transition is reached. In this respect the new model is again an improvement over the previous one (1) since that one shows an unphysical behavior in the baryon density as the chemical potential is increased. Another matrix model with improved thermodynamic properties was studied in [17], however, that model has not been solved for the eigenvalues.

At $\mu \geq 1$ the above scaling is no longer valid since the Gaussian integrals in the partition function would not converge. However, one can show that for $\mu>1$ and $\alpha>0$ the chiral condensate in the unquenched theory vanishes in the large $N$ limit. The model therefore does have a first order phase transition at $\mu=1$. Of course for large $\mu$ the Dirac operator should approach the deterministic form $\mu \gamma_{0}$ given in (1). Thus this new model cannot be expected to correctly describe the eigenvalue correlations in the phase where chiral symmetry is restored. It is currently then a phenomenological model for QCD at $\mu<\mu_{c}$ (here $\mu_{c}=1$ ).

Given this models success it would be interesting to include temperature into the model and study the full phase diagram as has done with previous matrix models [5]. A more complete analysis of the phase diagram will be saved for a later publication. Another interesting extension would be to make both $A$ and $B$ be random banded matrices with a power law decay like what was considered for $\mu=0$ in [18]. This was found to provide good agreement with an instanton liquid model for eigenvalue correlations at larger energies (beyond the Thouless energy). This also may provide an accurate way to study the finite temperature chiral restoration transition.

In conclusion we have introduced a new random matrix model for QCD with a baryon chemical potential that is based on the form of the Dirac operator in a chiral basis and is exactly solvable for all eigenvalue correlations. We have presented the correlations for any number of quark flavors and given results in the microscopic limit where they should agree with QCD due to universality. This new model also has a physically accurate thermodynamic behavior for $\mu<\mu_{c}$ and could provide a basis for a phenomenological model of the finite density QCD phase diagram.

I would like to thank G. Akemann and J.J.M. Verbaarschot for helpful discussions. This work was supported in part by NSF PHY 01-39929.

[1] F. Di Francesco, et. al., Int. J. Mod. Phys. A 9, 4257 (1994).

[2] P.J. Forrester and B. Jancovici, Int. J. Mod. Phys. A 11, 941 (1997).

[3] Y.V. Fyodorov and H.-J. Sommers, J. Phys. A 36, 3303 (2003).

[4] M.A. Stephanov, Phys. Rev. Lett. 76, 4472 (1996).

[5] M.A. Halasz, A.D. Jackson and J.J.M. Verbaarschot, Phys. Lett. B 395, 293 (1997); Phys. Rev. D 56, 5140 (1997); M.A. Halasz, et. al., Phys. Rev. D 58, 096007 (1998); B. Vanderheyden and A.D. Jackson, Phys. Rev. D 62, 094010 (2000); B. Klein, D. Toublan and J.J.M. Verbaarschot, Phys. Rev. D 68, 014009 (2003).

[6] M.A. Halasz, Nucl. Phys. A642, 324 (1998); M.A. Halasz, et.al., Phys. Rev. D 61, 076005 (2000); J. Ambjorn, et.al., JHEP 0210, 062 (2002).

[7] M.A. Halasz, J.C. Osborn and J.J.M. Verbaarschot, Phys. Rev. D 56, 7059 (1997).

[8] K. Splittorff and J.J.M. Verbaarschot, hep-th/0310271

[9] G. Akemann, Phys. Rev. Lett. 89, 072002 (2002); J. Phys. A 36, 3363 (2003).

[10] G. Akemann, Acta Phys. Polon. B 34, 4653 (2003).

[11] E.V. Shuryak and J.J.M. Verbaarschot, Nucl. Phys. A560, 306 (1993).

[12] Y.V. Fyodorov, B.A. Khoruzhenko and H.-J. Sommers, Phys. Lett. A 226 46, (1997).

[13] P.H. Damgaard and S.M. Nishigaki, Nucl. Phys. B518, 495 (1998).

[14] M.C. Bergere, hep-th/0311227

[15] T. Wilke, T. Guhr and T. Wettig, Phys. Rev. D 57, 6486 (1998).

[16] G. Akemann and G. Vernizzi, Nucl. Phys. B660, 532 (2003).

[17] M.A. Halasz, hep-lat/0011086

[18] A.M. Garcia-Garcia and J.C. Osborn, hep-th/0312146 